\title{
DNA methylation directly downregulates human cathelicidin antimicrobial peptide gene (CAMP) promoter activity
}

\author{
Xi Chen ${ }^{1, *}$, Guangying Qi ${ }^{2,4, *}$, Mingqun Qin ${ }^{3, *}$, Yantao Zou ${ }^{1}$, Kanghua Zhong ${ }^{1}$, Ying \\ Tang ${ }^{1}$, Yong Guo ${ }^{1}$, Xinxiang Jiang ${ }^{3}$, Lihua Liang ${ }^{3}$, Xianqiong Zou ${ }^{1}$ \\ ${ }^{1}$ College of Biotechnology, Guilin Medical University, Guilin 541100, Guangxi, P. R. China \\ ${ }^{2}$ Department of Pathology and Physiopathology, Guilin Medical University, Guilin 541004, Guangxi, P. R. China \\ ${ }^{3}$ Department of Stomatology, Affiliated Hospital of Guilin Medical University, Guilin 541004, Guangxi, P. R. China \\ ${ }^{4}$ Laboratory of Tumor Immunology and Microenvironmental Regulation, Guilin Medical University, Guilin 541004, Guangxi, \\ P. R. China \\ *These authors contributed equally to this work
}

Correspondence to: Xianqiong Zou, email: xianqiongzou2009@yahoo.com

Keywords: CAMP, LL-37, OSCC, DNA methylation, promoter

Received: September 01, $2016 \quad$ Accepted: February 20, $2017 \quad$ Published: March 02, 2017

Copyright: Chen et al. This is an open-access article distributed under the terms of the Creative Commons Attribution License (CC-BY), which permits unrestricted use, distribution, and reproduction in any medium, provided the original author and source are credited.

\section{ABSTRACT}

LL-37, the active product of human cathelicidin antimicrobial peptide (CAMP) has a broad spectrum of antibacterial activity. LL-37 also has important physiological functions in immune regulation, angiogenesis and in modulating apoptosis. The roles of LL-37 in oral squamous cell carcinoma (OSCC) are still not clear. The correlation between DNA methylation and human CAMP expression is also unknown. Here human CAMP/LL-37 expression was assessed by immunohistochemistry in normal and OSCC tissues. The results indicated that low expression of CAMP/LL-37 correlated with histological differentiation and lymph node metastasis and also promoted tumor progression. A cell-specific methylation pattern in the promoter region of human CAMP was detected. Treatment with 5-aza-2'-deoxycytidine, a DNA demethylation reagent can increase human CAMP expression in epithelial cancer cells. The reporter assay showed that unmethylated human CAMP promoter activity was significantly higher than methylated promoter activity. Taken together, these results suggested that human CAMP/LL-37 might act as a tumor-suppressor in OSCC and DNA methylation might play roles during carcinogenesis via directly downregulating human CAMP promoter activity.

\section{INTRODUCTION}

Keratinocytes are the first mucosal epithelial cells to provide barrier protection against both oral and ingested enteric pathogens [1]. Antimicrobial peptides (AMPs) within keratinocytes also appear to increase resistance to bacterial invasion $[1,2]$. Human cationic antibacterial peptide (CAMP, also called hCAP18) is the only cathelicidin in humans, and is primarily found in the secondary granules of neutrophils $[3,4]$. The C-terminal end of this protein also contains a 37-amino acid-long peptide (LL-37) with a broad-spectrum antibacterial activity $[4,5]$. Besides its broad antimicrobial activity,
LL-37 also plays roles in stimulation and modulation of cytokine release from different immune cells $[3,4]$. Recently, Overexpression of LL-37 was found to promote development and progression of ovarian, lung and breast cancers, and to suppress tumorigenesis in colon and gastric cancer [6, 7]. However, the roles of LL-37 in oral squamous cell carcinoma (OSCC) are not clear.

Human CAMP expression has been found in epithelial cells of the intestine, airway, genitals, ocular surface, skin and in eccrine glands [3]. In addition to its expression in epithelial tissues, cathelicidin is also produced by human neutrophils, Natural killer (NK) cells, mast cells, dendritic cells, monocytes and macrophages 
[3]. Human CAMP expression can be induced by 1,25-dihydroxyvitamin D3 $\left(1,25(\mathrm{OH})_{2} \mathrm{D}_{3}\right)$, Toll-like receptor agonists, injury and wounding, ER stress, sodium butyrate and TNFa $[3,4]$. Human CAMP expression can be downregulated by Bacterial exotoxins, Shigella infection, Psychological stress or Calcipotriol $[3,4]$. Transcription factors that can regulate human CAMP gene expression include cAMP-responsive element-binding protein (CREB) [3], Hypoxia inducible factor-1 (HIF-1) [4], Vitamin D receptor (VDR) [8], CCAAT enhancer binding protein $\alpha(\mathrm{C} / \mathrm{EBP} \alpha)[9]$, activator protein 1(AP-1) [10] and C/EBP $\beta$ [11]. ER stress increases CAMP

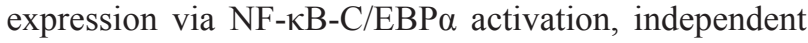
of VDR pathway [9]. Sodium butyrate up-regulates the CAMP gene via activator protein- 1 and histone acetylation at the promoter region in a human lung epithelial cell line, EBC-1 [10].

Gene expression can be regulated epigenetically by DNA methylation, histone variants and modifications and nucleosome positioning [12]. DNA methylation at $\mathrm{CpG}$ islands is associated with gene silencing $[13,14]$. In recent years it has been shown that DNA methylation at $\mathrm{CpG}$ sites in non- $\mathrm{CpG}$ island promoters can also contribute to regulate gene expression [12, 15-18]. Studies on transcriptional regulation of human $C A M P$ have been widely reported [3-11]. However, detailed mechanism about how DNA methylation regulates human $C A M P$ expression remains unclear. The purpose of this study is to explore roles of CAMP/LL-37 in OSCC and regulatory roles of DNA methylation in human CAMP promoter region.

\section{RESULTS}

\section{Expression of CAMP/LL-37 and Ki-67 in OSCC tissues}

To determine the cell proliferation activity and its association with CAMP expression we first compared the expression of CAMP/LL-37 and cellular proliferative activity marker Ki-67 in 70 OSCC and 20 normal tissues by immunohistochemistry. The results showed that CAMP/LL-37 is strongly expressed in normal oral mucosa (Figure 1A). However, it is weakly expressed in OSCC, especially in poorly-differentiated OSCC (Figure 1A). Ki-67 was only sparsely distributed in basal and parabasal layers in normal oral mucosa. However, Ki-67 is strongly expressed in OSCC (Figure 1A), while the low or high CAMP expression cases were 52/70 (74.3\%) and 18/70 (25.7\%), the low or high Ki-67 expression cases were $23 / 70(32.8 \%)$ and $47 / 70(67.2 \%)$, respectively (Figure 1A and Table 1). Moreover, among 52 OSCC with low CAMP/ LL-37 expression, 43 cases showed high expression of Ki67, whereas 18 cases showed high expression of CAMP/LL-37, and 14 cases showed low expression of Ki67 (Table 1). These results showed that CAMP/ LL-37 expression was negatively correlated with Ki-67 (Table 1). Subsequently, we examined the correlation between clinicopathological features and CAMP/LL-37 expression in OSCC. The low expression of CAMP/LL-37 was related to histological differentiation $(p<0.01)$ and lymph node metastasis $(p<0.01)$ in OSCC tissues, but was unrelated to age, gender and tumor size (Table 2).

\section{Low expression of CAMP in epithelial cancer cells}

To determine the expression levels of human CAMP in the epithelial cancer cell, HaCaT cell, TR146 cell and human oral cancer cell HSC-3, HSC-4, we then performed qRT-PCR and western blot analysis. The qRT-RCR analysis results showed that the mRNA copies of human $C A M P$ in these epithelial cancer cell lines are barely detectable (Figure 1B). The expression levels of human $C A M P$ are extremely low in these epithelial cancer cells compared with the positive control [TR146 cells treated with $1,25(\mathrm{OH})_{2} \mathrm{D}_{3}$ ]. However, the expression of human CAMP proteins in these epithelial cancer cells was also detectable (Figure 1C). Among these epithelial cancer cells, the expression levels of human CAMP in HaCaT cells are relatively high (Figure $1 \mathrm{C}$ ).

\section{Hypermethylation of CpG sites in human CAMP promoter}

The transcriptional regulation by human CAMP promoter has widely been reported [8-11]. Our bioinformatics analysis using CpGplot program indicated that the human $C A M P$ promoter is not located within $\mathrm{CpG}$ islands (http://www.ebi.ac.uk/Tools/seqstats/ emboss_cpgplot/). However, several CpG sites can be found at its promoter region (Figure 2A). To determine the methylation status of these $\mathrm{CpG}$ sites in human $C A M P$ promoter, bisulfite sequencing was performed. Because a product size larger than $300 \mathrm{bp}$ is very difficult to amplify [19], three short fragments containing CpG sites in human CAMP promoter were designed. The CAMP-F1 fragment (185 bp) has 2 CpG sites, CAMP-F2 fragment (172 bp) and CAMP-F3 fragment (179 bp) contains 5 and $2 \mathrm{CpG}$ sites, respectively. These fragments were then used for amplification. As shown in Figure 2, the methylation levels of $5 \mathrm{CpG}$ sites at CAMP-F2 in $\mathrm{HaCaT}$ and HSC4 cells were relatively high. In contrast, the methylation levels in TR146 and HSC-3 cells were relatively low. For TR146 and HSC-3 cells, the first and the fifth CpG sites with respect to the transcriptional direction at CAMP-F2 are fully unmethylated (Figure 2E). For HSC-4 cells, the first $\mathrm{CpG}$ sites at CAMP-F2 are fully unmethylated and most of the fifth $\mathrm{CpG}$ sites are methylated (Figure 2F). In contrast, the methylation levels for the $\mathrm{CpG}$ sites at 
Table 1: Correlation between human CAMP/LL-37 and Ki-67 expression in OSCC

\begin{tabular}{ccccc}
\hline & \multicolumn{2}{c}{ CAMP/LL-37 expression } & Total & p-value \\
\cline { 2 - 3 } & Low (52) & High (18) & & \\
\hline Ki-67 expression & 9 & 14 & 23 & $<0.01$ \\
Low & 43 & 4 & 47 & \\
High & & & & \\
\hline
\end{tabular}

Table 2: Human CAMP/LL-37 expression and its correlation with clinicopathological features in OSCC

\begin{tabular}{|c|c|c|c|}
\hline \multirow{3}{*}{ Clinicopathological features } & \multicolumn{3}{|c|}{ CAMP/LL-37 expression } \\
\hline & Low & High & $p$-value \\
\hline & 52 & 18 & \\
\hline \multicolumn{4}{|l|}{ Age (years) } \\
\hline$\geqq 50$ & 37 & 14 & \\
\hline$<50$ & 15 & 4 & \\
\hline \multicolumn{4}{|l|}{ Gender } \\
\hline Male & 35 & 13 & \\
\hline Female & 17 & 5 & \\
\hline \multicolumn{4}{|l|}{ Tumor size $(\mathrm{mm})$} \\
\hline$\geqq 15$ & 23 & 5 & \\
\hline$<15$ & 29 & 13 & \\
\hline \multicolumn{4}{|l|}{ Histological differentiation } \\
\hline Poor & 10 & 1 & \multirow{2}{*}{$<0.01$} \\
\hline $\mathrm{W} / \mathrm{M}^{\mathrm{a}}$ & 42 & 17 & \\
\hline \multicolumn{4}{|l|}{ Lymph node metastasis } \\
\hline Negative & 39 & 16 & \multirow{2}{*}{$<0.01$} \\
\hline Positive & 13 & 2 & \\
\hline
\end{tabular}

${ }^{a} \mathrm{~W} / \mathrm{M}$, Well or Moderately-differentiated OSCC.

CAMP-F1 and CAMP-F3 of all cells were low (Figure 2). The results indicated that the cell-specific methylation pattern in the promoter region of the human $C A M P$ may contribute to controlling its cell-specific expression. The results also suggested that the hypermethylation of CpG sites at CAMP-F2 in human CAMP promoter may participate into the inhibitory effects on the expression of human CAMP in these epithelial cancer cells.

\section{Induction of human CAMP expression by 5-aza- 2'-deoxycytidine (5-Aza-CdR) treatment}

To induce DNA demethylation, cells were incubated with 5-Aza-CdR (an inhibitor of DNA methylation) continuously for 6 days. After incubation, cells were collected for further analysis. Bisulfite sequencing (BSP) was also performed to confirm the demethylation effects of 5-Aza-CdR treatment. The results showed that 5-Aza-CdR treatment induced an obvious level of demethylation (Figure 3A). The 5-Aza-CdR treatment can significantly increase the expression of human
CAMP while the induction effects are moderate in these cell lines (Figure 3B and 3C). The induction effects for mRNA copies detected by qRT-PCR were not significant which might be caused by low levels of mRNA copies of $C A M P$ which are hard to accurately quantify in these cell lines (data not shown). These results indicated that other transcriptional regulators are also needed in these cell lines, and that DNA methylation in the promoter region of human $C A M P$ might downregulate expression of human CAMP.

\section{Direct downregulation of human CAMP gene promoter activity by DNA methylation}

Treatment of 5-Aza-CdR can induce genomewide demethylation, therefore the induction effects observed by 5 -Aza-CdR treatment might also be indirect (Figure 3). To clarify whether DNA methylation can directly downregulate human CAMP promoter activity, a pCpGfree-basic-Lucia construct devoid of $\mathrm{CpG}$ dinucleotides was used for luciferase reporter assay. 
pCpGfree-CAMP (-828/-1) was treated with M. SssI and M. HpaII methyltransferase. S-adenosylmethionine (SAM) was used as the methyl group donor for the enzymatic reaction. Plasmids of no treatment, M.SssI or SAM treatment were used as control. Methylated or unmethylated plasmids were then transfected into cells and luciferase activities were measured. The results indicated that a higher promoter activity was detected in these epithelial cancer cells transfected by unmethylated pCpGfree-CAMP (-828/-1) constructs. About 20-35\% lower promoter activity was found when $\mathrm{pCpGfree-CAMP}$ (-828/-1) constructs treated with M.SssI (methylated constructs) were transfected into HaCaT, TR146, HSC-3 or HSC-4 cells compared with unmethylated constructs (Figure 4). In addition, cells transfected with pCpGfreeCAMP (-828/-1) constructs that were only methylated by M. HpaII methyltransferase treatment also indicated a similar downregulation of promoter activity (Figure 4), which suggests that the $\mathrm{CpG}$ sites for $\mathrm{M}$. HpaII in human $C A M P$ promoter are important for the regulation while scattered methylation on $\mathrm{CpG}$ sites may not be effective to attenuate the gene promoter activity. The results obtained here also implied that the closest CpG site (-233/-232) to the major transcriptional start site of human CAMP is important for the transcriptional regulation (Figure 4). These observations are consistent with the results that the induction of human CAMP expression by 5 -Aza-CdR treatment (Figure 3). Taken together, these results strongly suggest that DNA methylation directly downregulates human CAMP promoter activity in these epithelial cancer cells.

\section{DISCUSSION}

Recently, LL-37 has been associated with tumor surveillance and anti-tumor effects [3, 6, 7]. Meanwhile, tumor-promoting effects have also been described $[3,6,7]$. The role of LL-37 in tumor development is tissue specific [6]. The expression levels of LL-37 are increased in ovarian, lung, breast cancer and malignant melanoma cells $[3,6]$. In contrast, the expression levels of LL-37 are decreased in cells from colon or gastric cancers $[3,6]$. Mechanistically, LL-37 contributes to
A
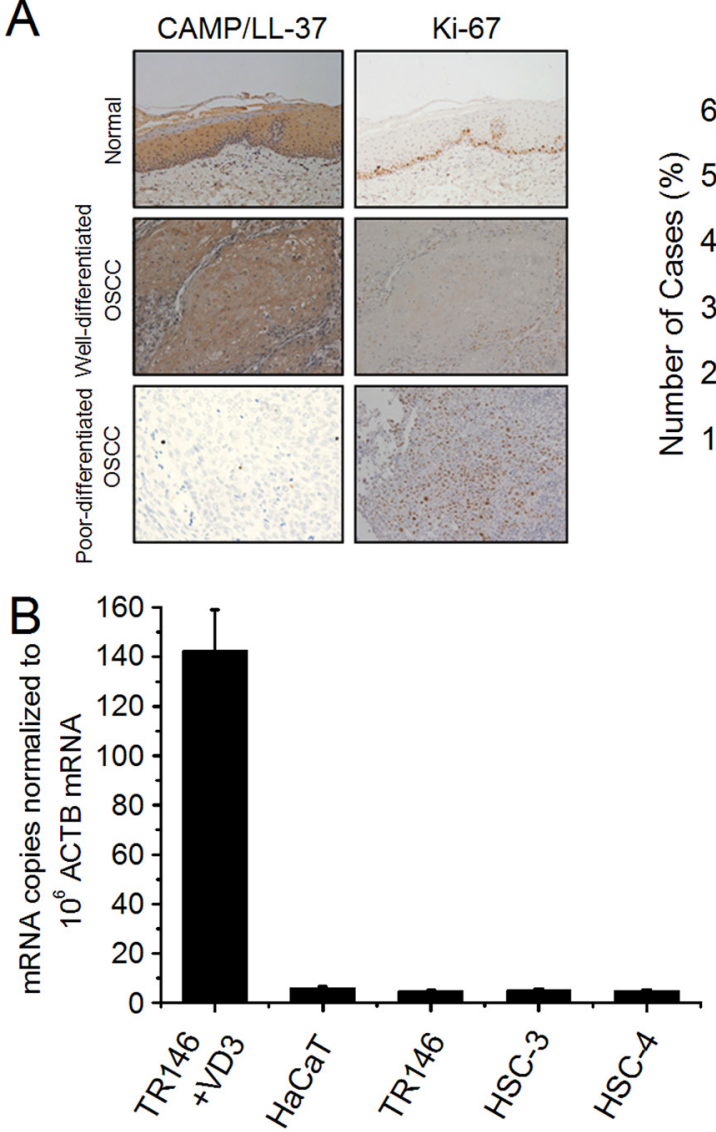

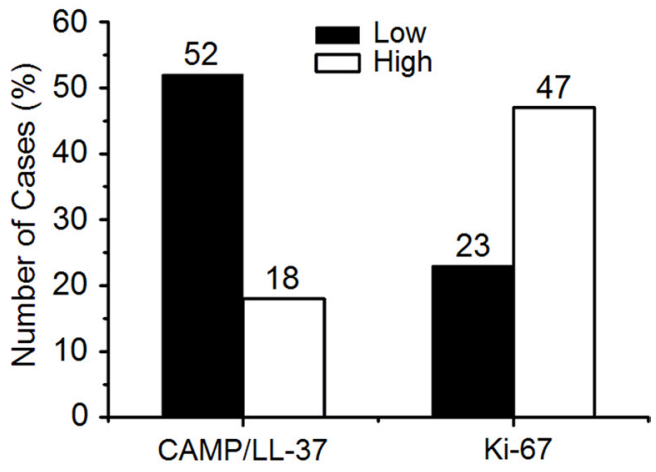

C

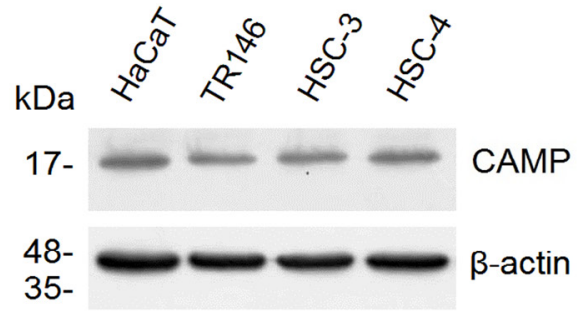

Figure 1: Expression analysis of human CAMP/LL-37 in normal oral mucosa, OSCC tissues and epithelial cancer cells. (A) Expression of human CAMP/LL-37 was examined by immunohistochemistry. Representative images of CAMP/LL-37 and Ki-67 in normal oral mucosa, well differentiated adenocarcinoma and poorly differentiated adenocarcinoma cases. (B) Human CAMP mRNA copies in cancer cells were detected by qRT-PCR, normalized to $\beta$-actin (ACTB) mRNA. TR146 cells treated with $1,25(\mathrm{OH})_{2} \mathrm{D}_{3}$ as described in materials and methods were used as positive control. $+\mathrm{VD} 3,1,25(\mathrm{OH})_{2} \mathrm{D}_{3}$ treatment. (C) Human CAMP protein expression in epithelial cancer cells was detected using western blot analysis. Intensity levels of each band are normalized to $\beta$-actin. The error bars show the means $\pm \mathrm{SE}$ of three to six independent experiments. 
A

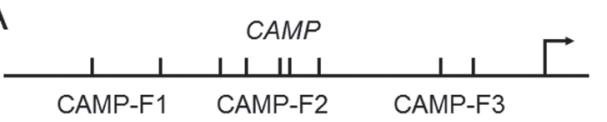

$\mathrm{B}$

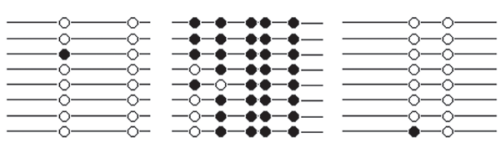

$\mathrm{C}=$

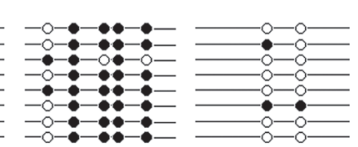

$\mathrm{D}$

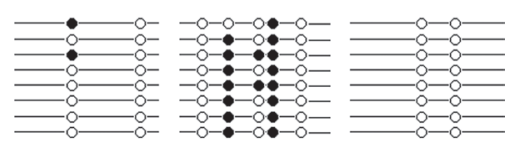

$E$

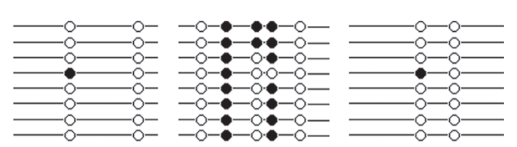

$\mathrm{F}$

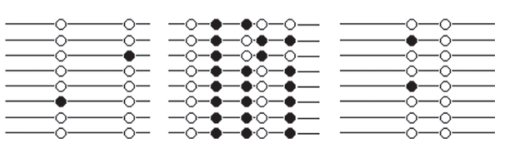

Figure 2: Cell specific methylation pattern for human CAMP. Bisulfite sequencing of CAMP-F1 fragment with 2 CpG sites (185 bp), CAMP-F2 fragment with 5 CpG sites (172 bp) and CAMP-F3 fragment with 2 CpG sites (179 bp) in human blood cells and epithelial cancer cells. At least eight independent clones were sequenced. Circles represent different $\mathrm{CpG}$ sites. Open and closed circles indicate unmethylated and methylated CpGs, respectively. (A) A schematic illustration of the human CAMP promoter. The major transcriptional start site is indicated by the arrow at position +1 . Upper tick marks indicate individual $\mathrm{CpG}$ sites in human CAMP promoter. (B) Methylation pattern in human peripheral blood cells. (C) Methylation pattern in HaCaT cells. (D) Methylation pattern in TR146 cells.

(E) Methylation pattern in HSC-3 cells. (F) Methylation pattern in HSC-4 cells.

A

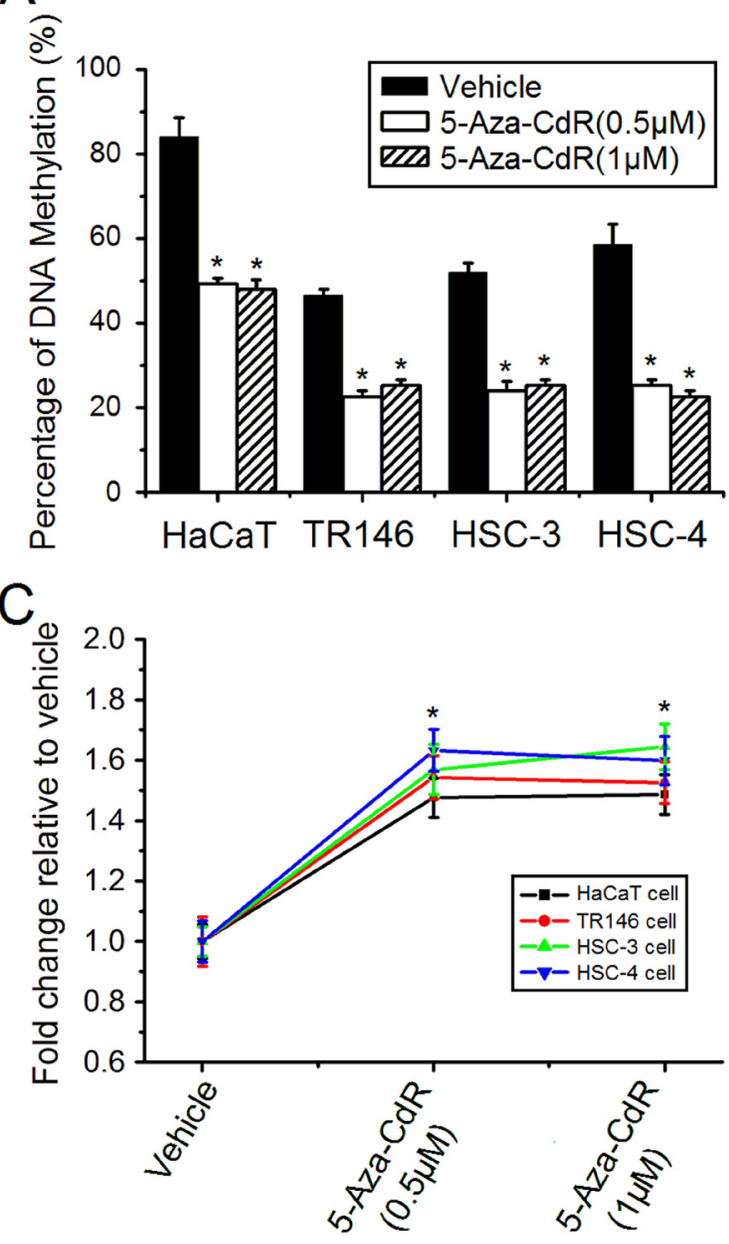

B

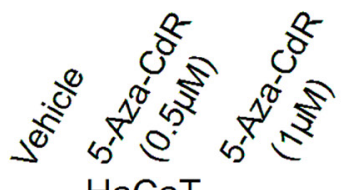

$\mathrm{HaCaT}$
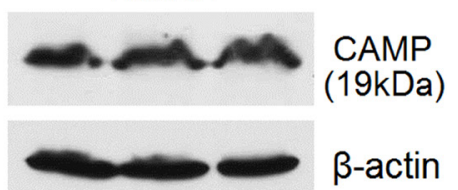

TR146

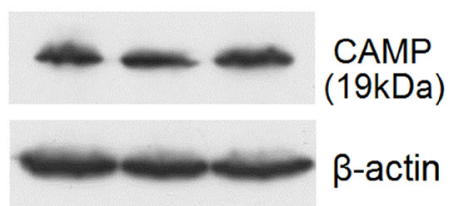

HSC-3

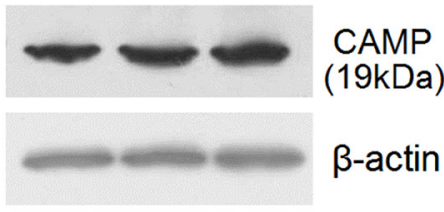

HSC-4

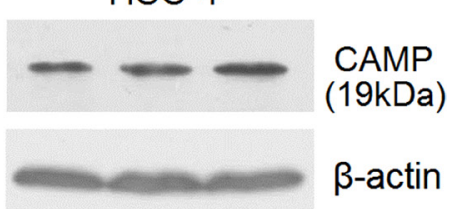

Figure 3: Induction of human CAMP expression by 5-Aza-CdR treatment. The expression levels of human CAMP protein were determined by western blot analysis. (A) The DNA methylation levels of CpG sites at CAMP-F2 in human CAMP promoter for the untreated cells and cells subjected to 5-Aza-CdR treatment were determined by bisulfite sequencing. At least five independent clones were sequenced each time. (B) Human CAMP protein was detected after 5-Aza-CdR treatment in epithelial cancer cells. (C) The expression levels of human CAMP protein were quantified after 5-Aza-CdR treatment. Intensity levels of each band are normalized to $\beta$-actin. The error bars show the means $\pm \mathrm{SE}$ of three to six independent experiments. * Significantly increased compared to vehicle control $(p<0.05)$. 
immunity, pathophysiology and cell signaling involved in malignant tumor growth $[6,20]$. Oral cancer is the sixth most common cause of death from cancer worldwide and the most common form of oral cancer is OSCC [21]. Little is known about the roles of LL-37 in OSCC. The immunohistochemistry results reported here indicated that the low expression cases of CAMP/LL-37 were $52 / 70(74.3 \%)$ in OSCC compared with normal oral mucosa (Figure 1A). The results also showed that the low expression of CAMP/LL-37 was related to histological differentiation, lymph node metastasis, and promoted tumor progression in OSCC tissues (Table 2). These results suggested that $\mathrm{CAMP} / \mathrm{LL}-37$ might act as a tumor suppressor in OSCC. Though the mechanism of CAMP/ LL-37 downregulation in OSCC is still not fully clear, for the first time the results reported here implied that CAMP/LL-37 might be useful for the development of a novel diagnostic marker and therapeutic target for OSCC patients.

Studies on transcriptional regulation of human $C A M P$ have been widely reported [3-11]. The epigenetic processes, such as DNA methylation, histone methylation and histone modification contribute to regulation of gene expression [12]. However, researches on a correlation between epigenetic processes and human CAMP expression have been limited so far [22-26]. Human colonic cancer cell lines HCT116 or Lovo were treated with different epigenetic modifying agents, 5-Aza-CdR, trichostatin A (TSA; a histone deacetylase inhibitor), and 3-deazaneplanocin A (DZNep; a histone methylation inhibitor). 5-Aza-CdR but not TSA or DZNep restored CAMP gene expression in cultured colonic cancer cells [22]. These results suggest that promoter DNA methylation was responsible for the downregulation of human CAMP gene expression in these human colonic cancer cell lines [22]. Histone modifications, such as methylation, acetylation are also involved in the gene regulation for human CAMP gene [23-26].

Due to the important roles of human CAMP/LL-37 in infectious diseases and tumors [1-7], the mechanism of transcriptional regulation of human $C A M P$ has attracted wide attention of researchers. DNA methylation has been functionally linked to gene repression [13]. It is known that the level of cytosine methylation of promoters is negatively correlated with gene expression [14, 27]. Here we explored the possible roles of DNA methylation in the promoter region for human CAMP expression. The results observed suggested that DNA methylation contributed to regulation of human CAMP expression and other transcription regulators also existed (Supplementary Figure 1). It was proposed that cytosine methylation might change the spatial structure of DNA and thus might affect transcriptional regulation by changes in the affinity of transcription factors binding to DNA [27-29]. Bioinformatics analysis using the TRANSFAC program indicated that methylation of the important $\mathrm{CpG}$ site at $-233 /-232$ could affect binding of transcription factors including GR-alpha, TFII-I, c-Jun and ER-alpha to human CAMP promoter. Though we couldn't detect methylation of $\mathrm{CpG}$ site at $-233 /-232$ of human $C A M P$ promoter in selected epithelial cancer cell lines, we did detect full methylation of $\mathrm{CpG}$ site at $-233 /-232$ human CAMP promoter in a colonic cancer cell line, Caco-2 cell (Supplementary Figure 2). According to results of reporter

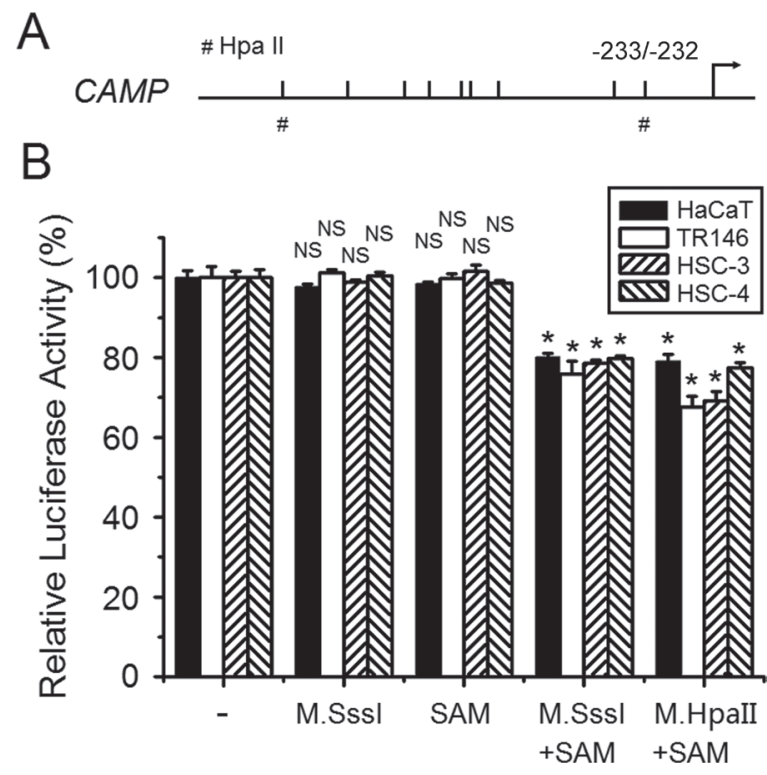

Figure 4: Direct downregulation of human CAMP promoter activity by DNA methylation. (A) A schematic illustration of the human $C A M P$ promoter. The major transcriptional start site is indicated by the arrow at position +1 . Upper tick marks indicate individual CpG sites in human CAMP promoter. The symbols of '\#' represent HpaII sites. (B) Luciferase activities of unmethylated and methylated human $C A M P$ promoter constructs in epithelial cancer cells. The error bars show the means $\pm \mathrm{SE}$ of three to six independent experiments. SAM, S-adenosylmethionine. NS, not significant. *Significantly decreased compared to untreated construct control $(p<0.05)$. 
assay, we expected that the CpG site at -233/-232 would play important roles for transcription regulation of human $C A M P$ in some specific cells, such as colonic cancer Caco-2 cells. Because hypermethylation of $\mathrm{CpG}$ sites at the CAMP-F2 fragment in human CAMP promoter could be detected in these oral epithelial cancer cells, TR146, HSC-3 and HSC-4 cells (Figure 2), we suggested that the transcription factors binding to these $\mathrm{CpG}$ sites at CAMP-F2 in human CAMP promoter might involve into the transcriptional regulation process in cancer progression for OSCC. Based on these results, we speculated that DNA methylation of these $\mathrm{CpG}$ sites in the promoter region might play important roles during carcinogenesis via directly downregulating human $C A M P$ promoter activity in cancer progression for OSCC. These investigations could be further confirmed using more oral cancer cell lines to have better understanding roles of DNA methylation of these $\mathrm{CpG}$ sites in cancer progression for OSCC. Further studies would also be necessary to clarify functional role of CAMP/LL-37 in oral cancer cells.

The investigation represents novel mechanistic insights into the epigenetic regulation of human $C A M P$ in oral epithelial cancer cells. Undoubtedly, the correlation established here between DNA methylation in the promoter region and human $C A M P$ expression will aid future elucidation of the regulatory mechanism of human $C A M P$ expression in physiological and pathological conditions.

\section{MATERIALS AND METHODS}

\section{Patients and tissue samples}

A total of 70 patients (ages 28 to 80 years, 48 men and 22 women) who had undergone surgery at the Affiliated Hospital of Guilin Medical University were enrolled in this study. All patients underwent complete surgical resection between 2009 and 2015. This study was approved by the Ethical Committees of Guilin Medical University and informed consent was obtained from all patients. Histologically, 59 cases were classified as well or moderately differentiated, with 11 classified as poorly differentiated OSCC. Clinical details including age, gender, tumor size, tumor differentiation and lymph node metastasis were gathered from surgical records of the patients. Tumors from each patient were formalinized and cut into parallel 4-5 $\mu \mathrm{m}$ sections.

\section{Immunohistochemical staining}

The sections were incubated with primary monoclonal anti-LL-37 antibody (1:200; Santa Cruz Biotechnology, sc-166770, Dallas, TX). In addition, to determine the proliferative cell activity and its correlate with CAMP/LL-37 expression, we examined Ki-67 expression using anti-Ki-67 monoclonal antibody (MIB-1, Dako, Carpinteria, CA). The sections were incubated with primary antibodies at $4^{\circ} \mathrm{C}$ overnight after antigen retrieval by microwave treatment in citrate buffer $(\mathrm{pH}$ 6.0), detection by the avidin-biotin peroxidase complex system using an LSAB kit (Doko, Kyoto, Japan). A labeling index, percentage of CAMP/LL-37 or Ki-67 positive cells was determined by examining of at least 1000 tumor cells at $200 \times$ magnification. The expression of CAMP/LL-37 and $\mathrm{Ki}-67$ were divided into high expression (more than $20 \%$ positive cells) and low expression (less than $20 \%$ positive cells).

\section{Cell culture}

Human HaCaT and TR146 cells were cultured in Dulbecco's Modified Eagle Medium (DMEM, Life technologies, Grand Island, NY) supplemented with 10\% Fetal Bovine Serum (FBS). HSC-3 and HSC-4 cells (human oral squamous cell carcinoma) were maintained in RPMI-1640 ((Life technologies, Grand Island, NY) supplemented with 10\% Fetal Bovine Serum (FBS). All cells were incubated at $37^{\circ} \mathrm{C}$ in a $5 \% \mathrm{CO}_{2}$ incubator. $\mathrm{HaCaT}$ cells were obtained from the American Type Culture Collection (ATCC, Manassas, USA). TR146 cells were provided by Dr. Xia Liu, Shandong Academy of Pharmaceutical Sciences, Jinan, P. R. China. HSC-3 and HSC-4 cells were obtained from Japanese Collection of Research Bioresources Cell Bank.

\section{Plasmids construction}

The PCR fragments of $-828 /-1$ of human $C A M P$ were amplified from human blood genomic DNA (Promega, Madison, WI) using primer pairs P1/P2 (Table 3) and Herculase ${ }^{\circledR}$ II Fusion DNA Polymerase (Agilent technologies, Santa Clara, CA). Amplified fragments were inserted into the pGEM-T vector (Promega, Madison, WI) to generate plasmid pGEM-T-828, which was verified by DNA sequencing. A 845 bp fragment amplified with primer pair P3/ P4 (Table 3) was ligated into a Lucia reporter plasmid pCpGfree-basic-Lucia (Invivogen, San Diego, CA) without a promoter and devoid of $\mathrm{CpG}$ dinucleotides via the AvrII and BamHI restriction sites to generate vector pCpGfree-CAMP (-828/-1). Constructs were confirmed by automated sequencing (Sangon, Shanghai, China).

\section{Bisulfite sequencing}

Genomic DNAs from HaCaT, TR146, HSC-3 and HSC-4 cells were isolated using the genomic DNA extraction kit (TianGen, Beijing, China), and then the purified genomic DNAs were bisulfite converted using the EZ DNA Methylation-Gold Kit (Zymo Research, Irvine, CA) according to the manufacturer's recommendations. Bisulfite-treated unmethylated DNA from human peripheral blood (Promega) was used as control. Platinium Taq polymerase (Life technologies) was 
Table 3: Oligonucleotides probes

\begin{tabular}{lcc}
\hline \multicolumn{1}{c}{ Name } & Oligonucleotide sequence $\left(\mathbf{5}^{\prime} \mathbf{- 3} \mathbf{3}^{\mathbf{a}} \mathbf{a}^{\mathbf{a}}\right.$ & Location $^{\mathbf{b}}$ \\
\hline Primers for Methylation analysis for CAMP & $-677 /-650$ \\
P-F1L & TGTTATTTAGGTTGGAGTGTAGTGGTAT & $-518 /-493$ \\
P-F1R & CAAACTTAACCAACATAATAAAACCC & $-524 /-501$ \\
P-F2L & GAGATGGGGTTTTATTATGTTGGT & $-377 /-353$ \\
P-F2R & CCAACTCTAAACATTACCTAACACC \\
P-F3L & ATTGATTTTTGAGGAGTAGAAGGAT & $-333 /-309$ \\
P-F3R & AAACAATAAATAAAACCTTCCTTATATACA & $-184 /-155$ \\
Primers for $p$ CpGfree-basic-Lucia constructs & $-828 /-807$ \\
P-1 & GTCTGGCTGACGGCTGGGTCCA \\
P-2 & TCCCTCTAGCCCACAGGAGCCTC & $-23 /-1$ \\
P-3 & CCACCTAGGGTCTGGCTGACGGCTGGGTCCA & $-828 /-807$ \\
P-4 & CGGGATCCTCCCTCTAGCCCACAGGAGCCTC & $-23 /-1$ \\
\hline
\end{tabular}

a Regions of oligonucleotide not derived from human CAMP are underlined.

${ }^{b}$ Oligonucleotide position is relative to major transcriptional start site of human $C A M P(=+1)$.

used for PCR amplification. Primer pairs P-F1L/P-F1R, P-F2L/P-F2R and P-F3L/P-F3R were used to amplify CAMP-F1, CAMP-F2 and CAMP-F3 fragments in human $C A M P$ promoter region, respectively (Table 3 ). Cycling conditions were: step $1,94^{\circ} \mathrm{C}$ for $3 \mathrm{~min}$; step $2,94^{\circ} \mathrm{C}$ for $30 \mathrm{sec}$; step $3,58^{\circ} \mathrm{C}$ for $30 \mathrm{sec}$; step $4,72^{\circ} \mathrm{C}$ for $30 \mathrm{sec}$; step 5, repeat step 2 to step 4 for 40 times; and step 6, extend at $72^{\circ} \mathrm{C}$ for $3 \mathrm{~min}$. Amplified fragments were separated on $1 \%$ agarose gel and purified, and then cloned into pGEM-T vectors (Promega) for DNA sequencing (Sangon, Shanghai).

\section{5-Aza-CdR treatment}

5-Aza-CdR (Sigma) was dissolved in 99\% ethanol to $10 \mathrm{mM}$, stored at $-20^{\circ} \mathrm{C}$ and used at a final concentration of $0.5 \mu \mathrm{M}$ or $1 \mu \mathrm{M}$ [30]. Treatment with 5-Aza-CdR was performed as described previously [30]. Cells were seeded at a density of 2 to $3 \times 10^{5}$ cells $/ \mathrm{ml}$. The medium was replaced every $24 \mathrm{~h}$ for 6 days with fresh medium containing $0.5 \mu \mathrm{M}$ or $1 \mu \mathrm{M} 5$-Aza-CdR, and then cells were collected for further analysis.

\section{CpG methyltransferase treatment and luciferase reporter assay}

The plasmid pCpGfree-CAMP (-828/-1) was treated with methyltransferase M. SssI or M. HpaII. Methylation was confirmed by treatment of restriction enzyme HpaII. Cells were seeded in 6-well plates. At approximately 60-90\% confluence, cells were transfected with plasmids methylated or unmethylated using Lipofectamine 3000 (Life technologies) according to the manufacturer's recommendations. Briefly, $2.5 \mu \mathrm{g}$ of plasmid DNA and
$5 \mu \mathrm{l}$ P3000 reagent was diluted in $125 \mu \mathrm{l}$ Opti-MEM and then were added into $125 \mu$ Opti-MEM with $5 \mu \mathrm{l}$ Liofectamine 3000 reagent. After 5 min of incubation, the mixture was added into each well. After $24 \mathrm{~h}$, medium was aspirated and replaced with $800 \mu \mathrm{l}$ fresh medium. $48 \mathrm{~h}$ after transfection, medium was collected and $20 \mu \mathrm{l}$ aliquots were added into 96 well plates. $100 \mu \mathrm{l}$ Quanti-Luc ${ }^{\mathrm{TM}}$ (Invivogen) was injected into each well and luciferase activities were read.

\section{Quantitative real-time polymerase chain reaction analysis (qRT-PCR)}

RNA was isolated using Trizol reagent (Life Technologies). Total RNA was reverse transcribed with FastQuant RT kit with gDNase (Tiangen). Quantitative Real-time Polymerase Chain Reaction (qRT-PCR) with SYBR was performed. Primers for qPCR amplification of human CAMP (FC1, CAAAGCCTGTGAGCTTCACAG. FC2, GGACTCTGTCCTGGGTACAAG) [31], and $A C T B$ (FA1, GACGACATGGAGAAAATCTG. FA2, ATGATCTGGGTCATCTTCTC) were used. TR146 cells treated with $10^{-8} \mathrm{M} 1,25(\mathrm{OH})_{2} \mathrm{D}_{3}$ (Sigma, St. Louis, MO) for $24 \mathrm{~h}$ were used as positive control [8]. The expression levels of human $C A M P$ were normalized to $\beta$-actin mRNA. The qRT-PCR results were processed using the $2^{-\Delta \Delta C t}$ method [32].

\section{Western blot analysis}

Cells were washed with Dulbecco's phosphatebuffered saline (DPBS, Gibco BRL, Life Technologies) and proteins were extracted using mammalian cell lysate buffer (Biyuntian, Beijing, China). Samples were then centrifuged 
at $14000 \mathrm{rpm}$ for $5 \mathrm{~min}$ at $4^{\circ} \mathrm{C}$ and protein concentrations of the supernatants were determined by the bicinchoninic acid (BCA) protein concentration detection kit (Biyuntian, Beijing, China). The extracted proteins were separated by $12 \%$ SDS-PAGE, transferred onto a $0.22 \mu \mathrm{M}$ nitrocellulose membranes, and incubated with mouse anti- $\beta$-actin (TA09, ZSGB-BIO, Beijing, China) or mouse anti-LL-37 (sc-166770, Santa Cruz Biotechnolology). Membranes were washed and then incubated with HRP-conjugated goat anti-mouse antibodies (EM35110, EMAR, Beijing, China). Immunoreactions were visualized using Pierce Western ECL substrate (Thermo Scientific, Rockford, IL) or Clarity ${ }^{\mathrm{TM}}$ Western ECL Substrate (BioRad, Hercules, CA) and exposed to Amersham Hyperfilm ECL film (GE Healthcare Biosciences, Piscataway, NJ). Protein bands were quantified by Quantity One analysis (BioRad).

\section{Statistical analysis}

Generally, statistical analysis was performed with SPSS 19.0 (IBM, Armonk, NY). Comparisons between two groups were done with Student's $t$ test, and multiple comparisons were done with one-way ANOVA followed by Bonferroni's post hoc test. For immunohistochemical staining analysis, the $\chi 2$ ant $T$ test was used for comparison of the data between two groups. A $p$ value less than 0.05 was considered to indicate statistical significance.

\section{ACKNOWLEDGMENTS}

We would like to thank Dr. Fred Bogott at Austin Medical Center, Austin of Minnesota, USA, and Dr. Joshua Liao at Hormel Institute, University of Minnesota, for their excellent English editing of the manuscript.

\section{CONFLICTS OF INTEREST}

No potential conflicts of interest were disclosed by all authors.

\section{GRANT SUPPORT}

This work was supported by grants from the National Natural Science Foundation of China (No. 81560185 ) and Natural Science Foundation of Guangxi (2015GXNSFCA139008).

\section{REFERENCES}

1. Sorenson BS, Khammanivong A, Guenther BD, Ross KF, Herzberg MC. IL-1 receptor regulates S100A8/A9dependent keratinocyte resistance to bacterial invasion. Mucosal Immunol. 2012; 5:66-75.

2. Gaffen SL, Herzberg MC, Taubman MA, Van Dyke TE.
Recent advances in host defense mechanisms/therapies against oral infectious diseases and consequences for systemic disease. Adv Dent Res. 2014; 26:30-37.

3. Vandamme D, Landuyt B, Luyten W, Schoofs LA. Comprehensive summary of LL-37, the factotum human cathelicidin peptide. Cell Immunol. 2012; 280:22-35.

4. Xhindoli D, Pacor S, Benincasa M, Scocchi M, Gennaro R, Tossi A. The human cathelicidin LL-37-A pore-forming antibacterial peptide and host-cell modulator. Biochim Biophys Acta. 2016; 1858:546-566.

5. Zou XQ, Sorenson BS, Ross KF, Herzberg MC. Augmentation of epithelial resistance to invading bacteria by using mRNA transfections. Infect Immun. 2013; 81:3975-3983.

6. Piktel E, Niemirowicz K, Wnorowska U, Wątek M, Wollny T, Głuszek K, Góźdź S, Levental I, Bucki R. The role of cathelicidin LL-37 in cancer development. Arch Immunol Ther Exp. 2016; 64:33-46.

7. Cheng M, Ho S, Yoo JH, Tran DH, Bakirtzi K, Su B, Tran DH, Kubota Y, Ichikawa R, Koon HW. Cathelicidin suppresses colonic cancer development by inhibition of cancer associated fibroblasts. Clin Exp Gastroenterol. 2014; $8: 13-29$.

8. Gombart AF, Borregaard N, Koeffler HP. Human cathelicidin antimicrobial peptide (CAMP) gene is a direct target of the vitamin $\mathrm{D}$ receptor and is strongly up-regulated in myeloid cells by 1,25-dihydroxyvitamin D3. FASEB J. 2005; 19:1067-1077.

9. Park K, Elias PM, Oda Y, Mackenzie D, Mauro T, Holleran WM, Uchida Y. Regulation of cathelicidin antimicrobial peptide expression by an endoplasmic reticulum (ER) stress signaling, vitamin D receptorindependent pathway. J Biol Chem. 2011; 286:34121-34130.

10. Kida Y, Shimizu T, Kuwano K. Sodium butyrate upregulates cathelicidin gene expression via activator protein-1 and histone acetylation at the promoter region in a human lung epithelial cell line, EBC-1. Mol Immunol. 2006; 43:1972-1981.

11. Svensson D, Nebel D, Nilsson BO. Vitamin D3 modulates the innate immune response through regulation of the hCAP-18/LL-37 gene expression and cytokine production. Inflamm Res. 2016; 65:25-32.

12. Han H, Cortez CC, Yang X, Nichols PW, Jones PA, Liang G. DNA methylation directly silences genes with non-CpG island promoters and establishes a nucleosome occupied promoter. Hum Mol Genet. 2011; 20:4299-4310.

13. Jones PA. Functions of DNA methylation: islands, start sites, gene bodies and beyond. Nat Rev Genet. 2012; 13:484-492.

14. Schübeler D. Function and information content of DNA methylation. Nature. 2015; 517:321-326.

15. Yamazaki J, Jelinek J, Lu Y, Cesaroni M, Madzo J, Neumann F, He R, Taby R, Vasanthakumar A, Macrae T, Ostler KR, Kantarjian HM, Liang S, et al. TET2 mutations affect non- 
cpg island dna methylation at enhancers and transcription factor-binding sites in chronic myelomonocytic leukemia. Cancer Res. 2015; 75:2833-2843.

16. Øster B, Linnet L, Christensen LL, Thorsen K, Ongen H, Dermitzakis ET, Sandoval J, Moran S, Esteller M, Hansen TF, Lamy P, COLOFOL steering group, Laurberg S, et al. Non-CpG island promoter hypomethylation and miR149 regulate the expression of SRPX2 in colorectal cancer. Int J Cancer. 2013; 132:2303-2315.

17. Hoivik EA, Witsoe SL, Bergheim IR, Xu Y, Jakobsson I, Tengholm A, Doskeland SO, Bakke M. DNA methylation of alternative promoters directs tissue specific expression of Epac2 isoforms. PLoS One. 2013; 8:e67925.

18. Naganuma K, Hatta M, Ikebe T, Yamazaki J. Epigenetic alterations of the keratin 13 gene in oral squamous cell carcinoma. BMC Cancer. 2014; 14:988.

19. Li LC. Designing PCR primer for DNA methylation mapping. Methods Mol Biol. 2007; 402:371-84.

20. Kuroda K, Okumura K, Isogai H, Isogai E. The human cathelicidin antimicrobial peptide 11-37 and mimics are potential anticancer drugs. Front Oncol. 2015; 5:144.

21. Johnson JJ, Miller DL, Jiang R, Liu Y, Shi Z, Tarwater L, Williams R, Balsara R, Sauter ER, Stack MS. Proteaseactivated Receptor-2 (PAR-2)-mediated Nf- $\kappa \mathrm{B}$ Activation Suppresses Inflammation-associated Tumor Suppressor MicroRNAs in Oral Squamous Cell Carcinoma. J Biol Chem. 2016; 291:6936-6945.

22. Ren SX, Cheng AS, To KF, Tong JH, Li MS, Shen J, Wong CC, Zhang L, Chan RL, Wang XJ, Ng SS, Chiu LC, Marquez VE, et al. Host immune defense peptide LL-37 activates caspase-independent apoptosis and suppresses colonic cancer. Cancer Res. 2012; 72:6512-6523.

23. Gschwandtner M, Zhong S, Tschachler A, Mlitz V, Karner S, Elbe-Bürger A, Mildner M. Fetal human keratinocytes produce large amounts of antimicrobial peptides: involvement of histone-methylation processes. J Invest Dermatol. 2014; 134:2192-2201.

24. Yedery RD, Jerse AE. Augmentation of cationic antimicrobial peptide production with histone deacetylase inhibitors as a novel epigenetic therapy for bacterial infections. Antibiotics. 2015; 4:44-61.
25. Liu Q, Liu J, Roschmann KI, van Egmond D, Golebski K, Fokkens WJ, Wang D, van Drunen CM. Histone deacetylase inhibitors up-regulate LL-37 expression independent of tolllike receptor mediated signalling in airway epithelial cells. J Inflamm. 2013; 10:15.

26. Canani RB, Costanzo MD, Leone L. The epigenetic effects of butyrate: potential therapeutic implications for clinical practice. Clinical Epigentics. 2012; 4:4.

27. Medvedeva YA, Khamis AM, Kulakovskiy IV, Ba-Alawi W, Bhuyan MS, Kawaji H, Lassmann T, Harbers M, Forrest AR, Bajic VB. FANTOM consortium. Effects of cytosine methylation on transcription factor binding sites. BMC Genomics. 2014;15:119.

28. Zhang X, Yang R, Jia Y, Cai D, Zhou B, Qu X, Han H, Xu L, Wang L, Yao Y, Yang G. Hypermethylation of Sp1 binding site suppresses hypothalamic POMC in neonates and may contribute to metabolic disorders in adults: impact of maternal dietary CLAs. Diabetes. 2014; 63:1475-1487.

29. Zelko IN, Mueller MR, Folz RJ. CpG methylation attenuates Sp1 and Sp3 binding to the human extracellular superoxide dismutase promoter and regulates its cell-specific expression. Free Radic Biol Med. 2010; 48:895-904.

30. Qiu H, Yashiro M, Shinto O, Matsuzaki T, Hirakawa K. DNA methyltransferase inhibitor 5-Aza-CdR enhances the radiosensitivity of gastric cancer cells. Cancer Sci. 2009; 100:181-188.

31. Chakraborty K, Maity PC, Sil AK, Takeda Y, Das S. cAMP stringently regulates human cathelicidin antimicrobial peptide expression in the mucosal epithelial cells by activating cAMP-response element-binding protein, AP-1, and inducible cAMP early repressor. J Biol Chem. 2009; 284:21810-21827.

32. Shaw AE, Reid SM, Ebert K, Hutchings GH, Ferris NP, King DP. Implementation of a one-step real-time RT-PCR protocol for diagnosis of foot-and-mouth disease. J Virol Methods. 2007; 143:81-85. 